\title{
Influence of experimental variables on thermally stimulated recovery results: analysis of simulations and real data on a polymeric system
}

\author{
NM Alves, ${ }^{1}$ JF Mano ${ }^{1 *}$ and JL Gómez Ribelles ${ }^{2}$ \\ ${ }^{1}$ Polymer Engineering Department, University of Minho, Campus of Azurém, 4800-058 Guimarães, Portugal \\ ${ }^{2}$ Centre for Biomaterials and Department of Applied Thermodynamics, Universidad Politécnica de Valencia, PO Box 22012, E-46071 \\ Valencia, Spain
}

\begin{abstract}
Thermally stimulated recovery (TSR) is a low frequency mechanical spectroscopy technique that allows investigation of conformational mobility in polymeric systems. In this study the effect of initial parameters chosen to perform experiments on the TSR response of a material in the thermal sampling mode is investigated. The studied experimental parameters are creep time $\left(t_{\sigma}\right)$ recovery time $\left(t_{\mathrm{r}}\right)$ and window width $\left(\Delta T_{\mathrm{w}}\right)$; all are independently changed at one constant creep temperature. A simple model, able to describe global TSR and TS measurements, is used to evaluate the influence of each of the different parameters. The simulations are conducted for a system with a uniform distribution of activation energies and a fixed pre-exponential factor. These simulation results are qualitatively compared with some experimental data obtained for semicrystalline poly(ethylene terephthalate) under different conditions in the glass transition region. The tendencies resulting from the influence of the studied parameters on the intensity, the position of the TS peaks and the corresponding activation energies are found to be the same for the experimental and simulated results. Only the variation of the activation energy with $t_{\sigma}$ is opposite to that observed with the modelling results; this feature is explained on the basis of structural relaxation effects.
\end{abstract}

(C) 2002 Society of Chemical Industry

Keywords: viscoelasticity; TSR; glass transition; distribution of characteristic times

\section{INTRODUCTION}

Thermally stimulated techniques, such as thermally stimulated depolarization currents (TSDC) ${ }^{1-5}$ and thermally stimulated recovery (TSR) ${ }^{6-10}$ use transient measurements during temperature scanning to monitor molecular mobility at relatively low frequencies and over a large temperature range. In such techniques the sample is subjected to static excitation (an electric field in TSDC and a stress in TSR) and is quenched to a sufficiently low temperature at which the characteristic times are much longer than the time-scale of the experiment. The response (polarization in TSDC and strain in TSR) is then read during controlled heating. The different relaxational processes are detected by sudden changes in the measured response. This occurs in certain temperature ranges, when the corresponding characteristic times of the processes are of the same magnitude as the time-scale of the experiment.

By using a specific experimental protocol (thermal sampling, TS, or thermal windowing), thermally stimulated techniques can be used to divide a complex process, characterized by a distribution of characteris- tic times, into its quasi-elementary components, enabling analysis of the fine structure of global spectra. ${ }^{3,6}$ Usually, the temperature dependence kinetics of each TS result are described by the Arrhenius equation, characterized by the two mean thermokinetic parameters: activation energy $E_{\mathrm{a}}$ and a pre-exponential factor $\tau_{0}$.

$$
\tau=\tau_{0} \exp \left(E_{\mathrm{a}} / R T\right)
$$

Here, $\tau$ is the retardation time and $R$ is the ideal gas constant.

A typical TS experiment is schematically explained in Fig 1 and may be described as follows: (1) a static stress $\sigma_{0}$ is applied at temperature $T_{\sigma}$ during a timeperiod $t_{\sigma}$ ( $T_{\sigma}$ varies from experiment to experiment in the region of the complex process); (2) the sample is quenched to $T_{\sigma}-\Delta T_{\mathrm{w}}\left(\Delta T_{\mathrm{w}}\right.$ is typically 3 or $\left.4^{\circ} \mathrm{C}\right)$ with the mechanical field applied; (3) the stress is removed and the mechanical strain is allowed to recover during a period of time $t_{\mathrm{r}}$ (typically $2-4 \mathrm{~min}$ ); (4) the sample is quenched to $T_{0}$, well below the temperature region of the global process (say $50^{\circ} \mathrm{C}$ below $T_{\sigma}$ ); (5) the strain

\footnotetext{
* Correspondence to: JF Mano, Polymer Engineering Department, University of Minho, Campus of Azurém, 4800-058 Guimarães, Portugal E-mail: jmano@dep.uminho.pt

Contract/grant sponsor: Fundação para a Ciência e Tecnologia; contract/grant number: PRAXIS/P/CTM/14171/1998

Contract/grant sponsor: Portuguese-Spanish Joint Research Action; contract/grant number: Spain: HP1999-0024; Portugal: E/69/00

(Received 17 July 2001; revised version received 12 November 2001; accepted 12 December 2001)
} 


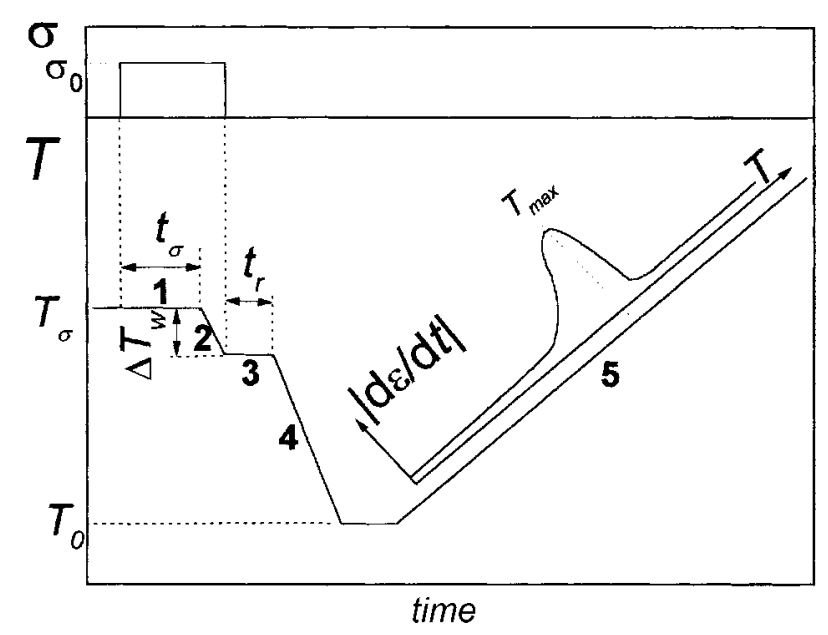

Figure 1. Schematic representation of a thermal sampling experiment. The static stress $\sigma_{0}$ is applied during steps 1 and 2. The release of the strain is measured during the heating process (step 5) as shown in the inset graphics showing a typical $\mathrm{d} \varepsilon / \mathrm{d} t$ vs $T$ plot.

is monitored during a controlled heating (typically at $4 \mathrm{~K} \mathrm{~min}^{-1}$ ), from $T_{0}$ up to a final temperature well above $T_{\sigma}$.

In TS experiments, the purpose is to excite as much as possible, a narrow distribution of characteristic times at a certain temperature from the broad spectrum of the entire process. It is possible to selectively fractionate the broad distribution of retardation times associated with the global process by performing different TS experiments at different $T_{\sigma}$ values in the region of the complex relaxation process under study. This resolution will depend on the experimental parameters used. In fact, one expects, for example, a better isolation of a narrow process if $\Delta T_{\mathrm{w}}$ is decreased. Other effects may arise if one changes $t_{\sigma}$ or $t_{\mathrm{r}}$. The modification of such parameters may also affect the thermokinetic parameters of the TS experiment. The simultaneous change of different parameters may complicate the final result even more. Although the discussion will be centred on TSR results, the main conclusions may be extended to the TSDC technique. The correct use of both TSR or TSDC techniques requires a good understanding and control of how the experimental parameters influence the final results, namely how they affect the distribution of retardation times isolated in a TS experiment and how the corresponding thermokinetic parameters are changed.

In this work, some TS results obtained for a semicrystalline polyethylene terephthalate (PET) film under different experimental conditions will be presented. To our knowledge, this systematic study has not been carried out either by TSR or by TSDC. Moreover, a simple model that is able to describe global TSR and TS experiments in equilibrated systems will be adapted from that used in the simulation of TSDC experiments ${ }^{11}$ and used to predict the effect of changing experimental parameters on TS experiments. The use of simulations allows one to work in 'clean' and 'controllable' systems, free of extraneous physical phenomena and experimental perturbations. Moreover, with simulations one can obtain information that is inaccessible by experiment, such as the distribution of characteristic times at any moment of the experiment.

\section{EXPERIMENTAL}

The material studied in this work was a semicrystalline PET obtained by crystallization of a commercial PET film supplied by Goodfellow (catalogue number ES301440). This material was annealed at $200^{\circ} \mathrm{C}$ for about $1 \mathrm{~h}$ before TSR experiments.

The TSR experiments were performed in a DMA7e Perkin-Elmer apparatus with a controlled cooling accessory. High purity helium was used for purging the sample environment during experiments, allowing better thermal contact between the furnace and the sample. More details of such experiments and data treatment may be found in papers by Alves and coworkers. $^{10,12}$ The use of a DMA apparatus offered the possibility of comparing dynamic mechanical analysis and TSR data obtained in the same equipment.

The experiments were carried out in the tensile mode. Rectangular samples $\left(0.18 \times 1.31 \mathrm{~mm}^{2}\right)$ were placed between two clamps. The bottom clamp was fixed and the top one applied static stress (in the case of this work $\sigma_{0}=18 \mathrm{MPa}$ ). In all experiments the heating rate in step 5 was $\beta=\mathrm{d} T / \mathrm{d} t=4^{\circ} \mathrm{C} \mathrm{min}{ }^{-1}$ and in both steps 2 and 4 the cooling rate was $\beta=-10^{\circ} \mathrm{C}$ $\min ^{-1}$. All experiments were carried out at $T_{\sigma}=93^{\circ} \mathrm{C}$. The experimental parameters changed in this work were the stress time $t_{\sigma}$, the temperature window width $\Delta T_{\mathrm{w}}$ and the recovery time $t_{\mathrm{r}}$.

\section{MODELLING OF THERMAL SAMPLING EXPERIMENTS}

Each TS experiment may be analysed as the mechanical relaxation of an elementary process characterized by a single retardation time $\tau$. The dependence of the strain $\varepsilon$ upon time, temperature and stress may be given by the Voigt-Kelvin model:

$$
\tau(T) \frac{\mathrm{d} \varepsilon(t)}{\mathrm{d} t}=\frac{\sigma_{0}}{E}-\varepsilon(t)
$$

where $\sigma_{0}$ is the static strain and $E$ is the Young modulus of the spring element (note that $\sigma_{0} / E$ is the strain in a creep experiment at $\left.t \rightarrow \infty, \varepsilon_{\infty}\right)$. Obviously, the majority of real systems cannot be quantified according to a single retardation time. The simplest way to consider this fact is to assume a distribution of uncoupled activated Voigt-Kelvin processes. The total strain at a certain time should be calculated taking into account a distribution of retardation times, described by a normalized probability density function, $\rho(\ln \tau)$ :

$$
\varepsilon(t)=\int_{-\infty}^{+\infty} \varepsilon(\tau, t) \rho(\ln \tau) \mathrm{d} \ln \tau
$$


where $\varepsilon(\tau, t)$ is the strain of the elementary process with a retardation time $\tau$.

To compute the strain of each individual molecular process during the TS experiment (step 5 in Fig 1) one needs to solve the differential eqn (2) for all steps (1-5). The total strain during step 5 is obtained from eqn (3).

The simulation of TS experiments requires the definition of $\rho(\ln \tau)$. Classic examples classify such distributions according to the distribution of the Arrhenius parameters. ${ }^{13}$ In those cases a distribution of retardation may arise either from (i) a distribution of activation energies ( $\tau_{0}$ is fixed), (ii) a distribution of pre-exponential factors ( $E_{\mathrm{a}}$ is fixed) or (iii) a distribution combining both parameters. A typical occurrence of the last distribution arises from a linear relationship between $E_{\mathrm{a}}$ and $\ln \tau_{0}$. The thermokinetic parameters obtained in the glass transition of polymers or other glass-forming materials always show this characteristic. Simulations considering all three possibilities have been investigated before, with the corresponding comparison with experimental results. ${ }^{14}$

An example is given here for the description of the simulation of TS experiments on a system characterized by an activation energy distribution, with a probability density function $g\left(E_{\mathrm{a}}\right)$. The solutions of eqns (2) and (3) for step 1 in Fig 1 are as follows:

$$
\begin{aligned}
& \varepsilon_{1}\left(E_{\mathrm{a}}\right)=\varepsilon_{\infty}\left(T_{\sigma}\right)\left[1-\exp \left(-\frac{t_{\sigma}}{\tau\left(E_{\mathrm{a}}, T_{\sigma}\right)}\right)\right] \\
& \varepsilon_{1}=\varepsilon_{\infty}\left(T_{\sigma}\right)\left[1-\int_{0}^{\infty} \exp \left(-\frac{t_{\sigma}}{\tau\left(E_{\mathrm{a}}, T_{\sigma}\right)}\right) g\left(E_{\mathrm{a}}\right) \mathrm{d} E_{\mathrm{a}}\right]
\end{aligned}
$$

Equation (4a) describes the strain at the end of step 1 (see Fig 1) for an elementary process of activation energy $E_{\mathrm{a}}$. Equation (4b) estimates the total strain at the end of step 1. $\tau\left(E_{\mathrm{a}}, T_{\sigma}\right)$ is calculated with eqn (1). It is assumed that $\varepsilon_{\infty}$ is independent of $E_{\mathrm{a}}$ and of temperature between $T_{\sigma}-\Delta T_{\mathrm{w}}$ and $T_{\sigma}$. The introduction of the dependence in $E_{\mathrm{a}}$ is implicit in $g\left(E_{\mathrm{a}}\right)$, the normalized probability function of $E_{\mathrm{a}}$ (ie $\left.\int_{0}^{\infty} g\left(E_{\mathrm{a}}\right) \mathrm{d} E_{\mathrm{a}}=1\right)$. The individual and global strains after step 2 are

$$
\begin{aligned}
\varepsilon_{2}\left(E_{\mathrm{a}}\right)= & \left(\varepsilon_{1}\left(E_{\mathrm{a}}\right)-\varepsilon_{\infty}\left(T_{\sigma}\right)\right) \exp \left[-\frac{1}{\beta_{1}} \int_{T_{\sigma}}^{T_{\sigma}-\Delta T_{\mathrm{w}}} \frac{\mathrm{d} T}{\tau\left(E_{\mathrm{a}}, T\right)}\right] \\
& +\varepsilon_{\infty}\left(T_{\sigma}\right)
\end{aligned}
$$

and

$$
\begin{aligned}
\varepsilon_{2}= & \varepsilon_{\infty}\left(T_{\sigma}\right)+\int_{0}^{\infty}\left(\varepsilon_{1}\left(E_{\mathrm{a}}\right)\right. \\
& \left.-\varepsilon_{\infty}\left(T_{\sigma}\right)\right) \exp \left[-\frac{1}{\beta_{1}} \int_{T_{\sigma}}^{T_{\sigma}-\Delta T_{\mathrm{w}}} \frac{\mathrm{d} T}{\tau\left(E_{\mathrm{a}}, T\right)}\right] g\left(E_{\mathrm{a}}\right) \mathrm{d} E_{\mathrm{a}}
\end{aligned}
$$

The individual and global strains after step 3 are

$$
\varepsilon_{3}\left(E_{\mathrm{a}}\right)=\varepsilon_{2}\left(E_{\mathrm{a}}\right) \exp \left(-\frac{t_{\mathrm{r}}}{\tau\left(E_{\mathrm{a}}, T_{\sigma}-\Delta T_{\mathrm{w}}\right)}\right)
$$

and

$$
\varepsilon_{3}=\int_{0}^{\infty} \varepsilon_{2}\left(E_{\mathrm{a}}\right) \exp \left(-\frac{t_{\mathrm{r}}}{\tau\left(E_{\mathrm{a}}, T_{\sigma}-\Delta T_{\mathrm{w}}\right)}\right) g\left(E_{\mathrm{a}}\right) \mathrm{d} E_{\mathrm{a}}
$$

The individual and global strains at the end of step 4 are

$$
\varepsilon_{4}\left(E_{\mathrm{a}}\right)=\varepsilon_{3}\left(E_{\mathrm{a}}\right) \exp \left[-\frac{1}{\beta_{3}} \int_{T_{\sigma}-\Delta T_{\mathrm{w}}}^{T_{0}} \frac{\mathrm{d} T}{\tau\left(E_{\mathrm{a}}, T\right)}\right]
$$

and

$$
\varepsilon_{4}=\int_{0}^{\infty} \varepsilon_{3}\left(E_{\mathrm{a}}\right) \exp \left[-\frac{1}{\beta_{3}} \int_{T_{\sigma}-\Delta T_{\mathrm{w}}}^{T_{0}} \frac{\mathrm{d} T}{\tau\left(E_{\mathrm{a}}, T\right)}\right] g\left(E_{\mathrm{a}}\right) \mathrm{d} E_{\mathrm{a}}
$$

Note that eqns (7a), (b) are of particular importance because they define the strain of the sample just before the final heating rate. In fact, the isothermal stage at $T_{0}$ was not considered because the retardation times of the stored processes are assumed to be much higher than the time stage at $T_{0}$, and thus no recovery occurs at $T_{0}$.

During the final heating rate, the strains are temperature dependent:

$$
\varepsilon_{5}\left(E_{\mathrm{a}}, T\right)=\varepsilon_{4}\left(E_{\mathrm{a}}\right) \exp \left[-\frac{1}{\beta} \int_{T_{0}}^{T} \frac{\mathrm{d} T^{\prime}}{\tau\left(E_{\mathrm{a}}, T^{\prime}\right)}\right]
$$

and

$$
\varepsilon_{5}(T)=\int_{0}^{\infty} \varepsilon_{4}\left(E_{\mathrm{a}}\right) \exp \left[-\frac{1}{\beta} \int_{T_{0}}^{T} \frac{\mathrm{d} T^{\prime}}{\tau\left(E_{\mathrm{a}}, T^{\prime}\right)}\right] g\left(E_{\mathrm{a}}\right) \mathrm{d} E_{\mathrm{a}}
$$

Equations (4)-(8) are easily adapted for the cases of both a distribution of pre-exponential factor and the occurrence of a compensation phenomenon. ${ }^{11}$

\section{SIMULATION RESULTS AND DISCUSSION}

The simulated results of this work were obtained for a system with a uniform distribution of activation energies, bracketed between $E_{1}=58 \mathrm{kJmol}^{-1}$ and $E_{2}=154.4 \mathrm{~kJ} \mathrm{~mol}^{-1}$ and a fixed pre-exponential factor $\tau_{0}=10^{-10} \mathrm{~s}$. Therefore, the probability density function $g\left(E_{\mathrm{a}}\right)$ is given by $1 /\left(E_{2}-E_{1}\right)$ if $E_{1}<E_{\mathrm{a}}<E_{2}$ and zero elsewhere. This distribution was chosen because some simulations of global and TS experiments at different polarization temperatures had already been analysed by TSDC. ${ }^{11}$ The following parameters were used in this work (the same as in the work of Mano ${ }^{11}$ : $\beta_{1}=\beta_{3}=-20^{\circ} \mathrm{C} \mathrm{m^{-1 }}$ (cooling rates of steps 2 and 4 ) and $\beta=4{ }^{\circ} \mathrm{C} \min ^{-1}$ (heating rate in step 5). In the present study only one creep temperature was used: $T_{\sigma}=177^{\circ} \mathrm{C}$. Unless otherwise stated, $t_{\sigma}=10 \mathrm{~min}$, 
$\Delta T_{\mathrm{w}}=3^{\circ} \mathrm{C}$ and $t_{\mathrm{r}}=2 \mathrm{~min}$ were also used. Simulated results were computed by changing these three variables independently. Figure 2 shows some such simulations.

Figure 2(a) shows the enlargement of the peaks as $t_{\sigma}$ increases. This should be expected because when this parameter increases the total creep increases in step 1. This increase of creep is mainly a consequence of a progressive activation of higher retardation times at $T_{\sigma}$. Preferentially, the creep assigned to such times will be kept during the recovery process in step 3 and will be recovered during step 5 at higher temperatures. This explains the increase of the area of such peaks (which is proportional to the creep stored at the end of step 4) and the shift of the peaks to higher temperatures as $t_{\sigma}$ increases.

The influence of the temperature window width $\Delta T_{\mathrm{w}}$ of step 2 also influences the final result (Fig 2(b)). As $\Delta T_{\mathrm{w}}$ increases, the distribution of retardation times activated at $T_{\sigma}$ and then modified by the jump to $T_{\sigma}-\Delta T_{\mathrm{w}}$ is shifted to lower time-values; therefore the recovery at $T_{\sigma}-\Delta T_{\mathrm{w}}$ will be less intense, especially on the lower side of the distribution of retardation times. Therefore an intensification of the TS peaks should be expected together with a corresponding shift to lower temperatures as $\Delta T_{\mathrm{w}}$ increases. This is, in fact, what is observed in the simulated results (Fig 2(b)).

Finally, let us analyse the influence of the recovery time $t_{\mathrm{r}}$ at step 3. When this parameter increases, the recovery of the remaining strain at the end of step 2 takes longer. Thus, a decrease of the area of the final TS peaks is expected. Moreover, as $t_{\mathrm{r}}$ increases, the recovery process will proceed faster in the strained elements with shorter retardation times. Therefore the remaining elements will be relatively enriched in slower processes, and will recover in step 5 at higher temperatures. This prediction is clearly observed in Fig 2(c) which shows a shift of the peaks to higher temperatures as $t_{\mathrm{r}}$ increases.

The temperature dependence of the retardation time of the TS experiments recorded in step 5 can be calculated from the following equation

$$
\tau(T)=-\frac{1}{\beta} \frac{\varepsilon(T)}{\mathrm{d} \varepsilon(T) / \mathrm{d} T}
$$

which was obtained by differentiation of eqn (8a). More details on the use of eqn (9) are discussed by Alves and co-workers. ${ }^{10,12}$ As an example, the Arrhenius lines of the TS curve in Fig 2(c) are shown in Fig 3. The curves are quite straight except for higher temperatures because a TS experiment does not perfectly resolve a complex process, especially near and above the maximum peak temperature. ${ }^{11}$

Because it is impossible to completely individualize a TS peak with a unique retardation time, the strain at the beginning of step 5 is associated with a distribution of retardation times (in the studied case, a distribution of activation energies). Figure 4 shows the distribution of activation energies that contributes to the total strain at the beginning of step 5 for all the cases shown in Fig 2. This distribution is presented in terms of $\varepsilon_{4}\left(E_{\mathrm{a}}\right) g\left(E_{\mathrm{a}}\right)$. The total area of each peak corresponds to the total strain at the end of step 4 . Note that such information cannot be directly obtained from experimental data.

The position of the distribution peaks in Fig 4 are in accordance with the TS peaks in Fig 2 . In fact, when the increase of the experimental parameter leads to a shift to higher temperatures of the TS peaks, we then
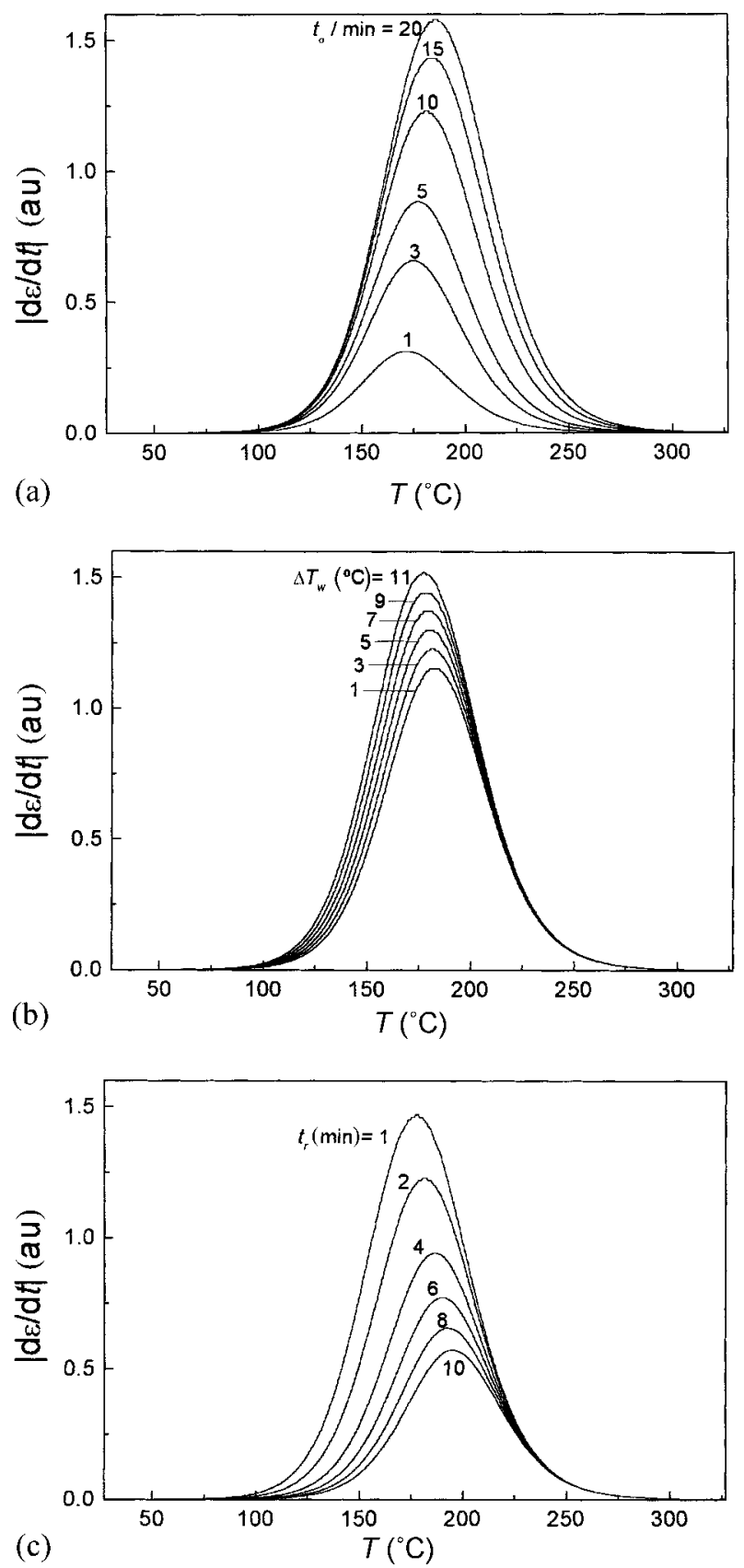

Figure 2. Dependence of simulated TS curves (creep rate against temperature) of a system characterized by a uniform distribution of activation energies between 58 and $154.4 \mathrm{~kJ} \mathrm{~mol}^{-1}$ and $\tau_{0}=10^{-10} \mathrm{~s}$ at $177^{\circ} \mathrm{C}$. Common experimental parameters: $\beta_{1}=\beta_{3}=-20^{\circ} \mathrm{C} \mathrm{min}{ }^{-1}$ and $\beta=4^{\circ} \mathrm{C}$ min $^{-1}$ : (a) Influence of the creep time $t_{\sigma}$, with $\Delta T_{\mathrm{w}}=3^{\circ} \mathrm{C}$ and $t_{\mathrm{r}}=2 \mathrm{~min}$; (b) influence of the window width $\Delta T_{\mathrm{w}}$, with $t_{\sigma}=10 \mathrm{~min}$ and $t_{\mathrm{r}}=2 \mathrm{~min}$; (c) influence of the recovery time, $t_{\mathrm{r}}$, with $\Delta T_{\mathrm{w}}=3^{\circ} \mathrm{C}$ and $t_{\sigma}=10 \mathrm{~min}$. 


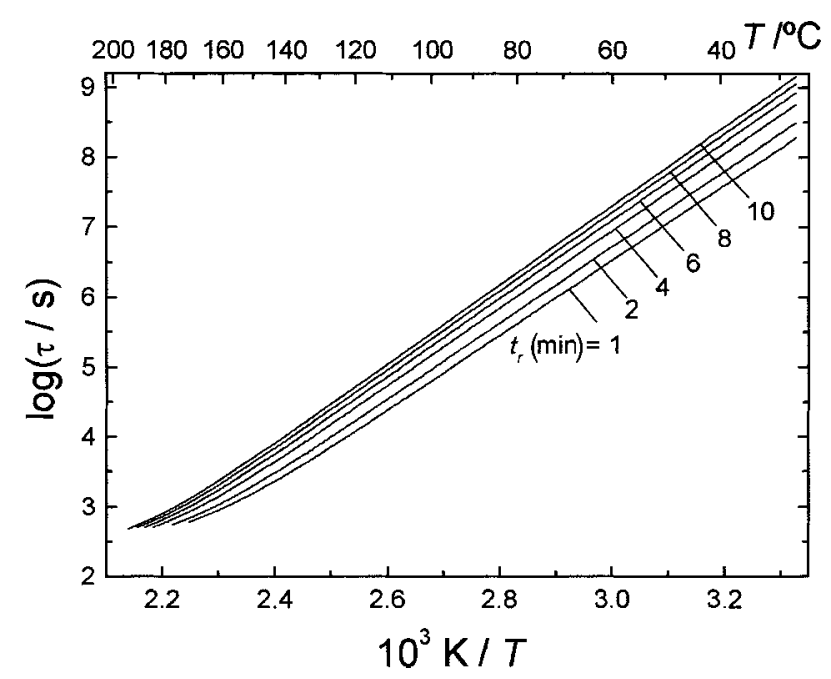

Figure 3. Arrhenius lines corresponding to the TS results shown in Fig 2(c), calculated using eqn (9).

have richer processes with higher activation energies, ie the corresponding distribution peaks should shift to higher $E_{\mathrm{a}}$. This is observed with the effects of increasing $t_{\sigma}$ and $t_{\mathrm{r}}$ and the opposite is observed with the effect of increasing $\Delta T_{\mathrm{w}}$.

The narrowing of the distribution of activation energies may be achieved by changing the experimental variables. This effect may be quantified by looking at the mid-height width $\Delta E_{\mathrm{a}, 1 / 2}$ of the distribution peaks. The better the isolation, the lower should be $\Delta E_{\mathrm{a}, 1 / 2}$. For the experiments shown in Fig 2 (a), at $t_{\sigma}=1,3,5,10,15$ and $20 \mathrm{~min}$, the obtained $\Delta E_{\mathrm{a}, 1 / 2}$ values are, respectively, $9.3 \mathrm{~kJ} \mathrm{~mol}^{-1}, 9.6 \mathrm{~kJ}$ $\mathrm{mol}^{-1}, 9.7 \mathrm{~kJ} \mathrm{~mol}^{-1}, 10.3 \mathrm{~kJ} \mathrm{~mol}^{-1}, 10.8 \mathrm{~kJ} \mathrm{~mol}^{-1}$ and $11.4 \mathrm{~kJ} \mathrm{~mol}^{-1}$. Thus, one can obtain well isolated TS experiments by decreasing the creep time. For the experiments shown in Fig 2(b), with $\Delta T_{\mathrm{w}}=1,3,5,7,9$ and $11^{\circ} \mathrm{C}$, the $\Delta E_{\mathrm{a}, 1 / 2}$ values are, respectively, $10.2 \mathrm{~kJ} \mathrm{~mol}^{-1}, 10.3 \mathrm{~kJ} \mathrm{~mol}^{-1}, 10.4 \mathrm{~kJ} \mathrm{~mol}^{-1}, 10.7 \mathrm{~kJ}$ $\mathrm{mol}^{-1}, 10.8 \mathrm{~kJ} \mathrm{~mol}^{-1}$ and $11.0 \mathrm{~kJ} \mathrm{~mol}^{-1}$. Therefore, the peaks are better isolated as $\Delta T_{\mathrm{w}}$ decreases, as expected. However, this effect is much smaller than the influence of $t_{\sigma}$. Finally, the experiments at different recovery times (Fig 2(c)), with $t_{\mathrm{r}}=1,2,4,6,8$ and $10 \mathrm{~min}$ yield $\Delta E_{\mathrm{a}, 1 / 2}$ values of $10.9 \mathrm{~kJ} \mathrm{~mol}^{-1}, 10.4 \mathrm{~kJ}$ $\mathrm{mol}^{-1}, 9.9 \mathrm{~kJ} \mathrm{~mol}^{-1}, 9.6 \mathrm{~kJ} \mathrm{~mol}^{-1}, 9.5 \mathrm{~kJ} \mathrm{~mol}^{-1}$ and $9.4 \mathrm{~kJ} \mathrm{~mol}^{-1}$. It can be concluded that, for the case of a system characterized by a distribution of activation energies, a better isolation of quasi-elementary TS peaks may be achieved by decreasing $t_{\sigma}$ instead of, for example, decreasing $\Delta T_{\mathrm{w}}$. However, the decrease of $t_{\sigma}$ leads to a reduction of the intensity of the peaks much faster than the decrease of $\Delta T_{\mathrm{w}}$.

\section{EXPERIMENTAL RESULTS}

Experimental data were collected using a similar strategy to that employed for the simulated results: we fixed the creep temperature and varied indepen- dently $t_{\sigma}, \Delta T_{\mathrm{w}}$ and $t_{\mathrm{r}}$. Some of these results, from experiments carried out at $T_{\sigma}=93^{\circ} \mathrm{C}$ on the studied PET sample, are shown in Fig 5. It is interesting to note that the tendencies related to the influence of the studied experimental parameters on the intensity of the peaks and on their position in the temperature axis are the same for the experimental and simulated results. In fact, as $t_{\sigma}$ increases the intensity of the peaks increases and the maximum temperature shifts to
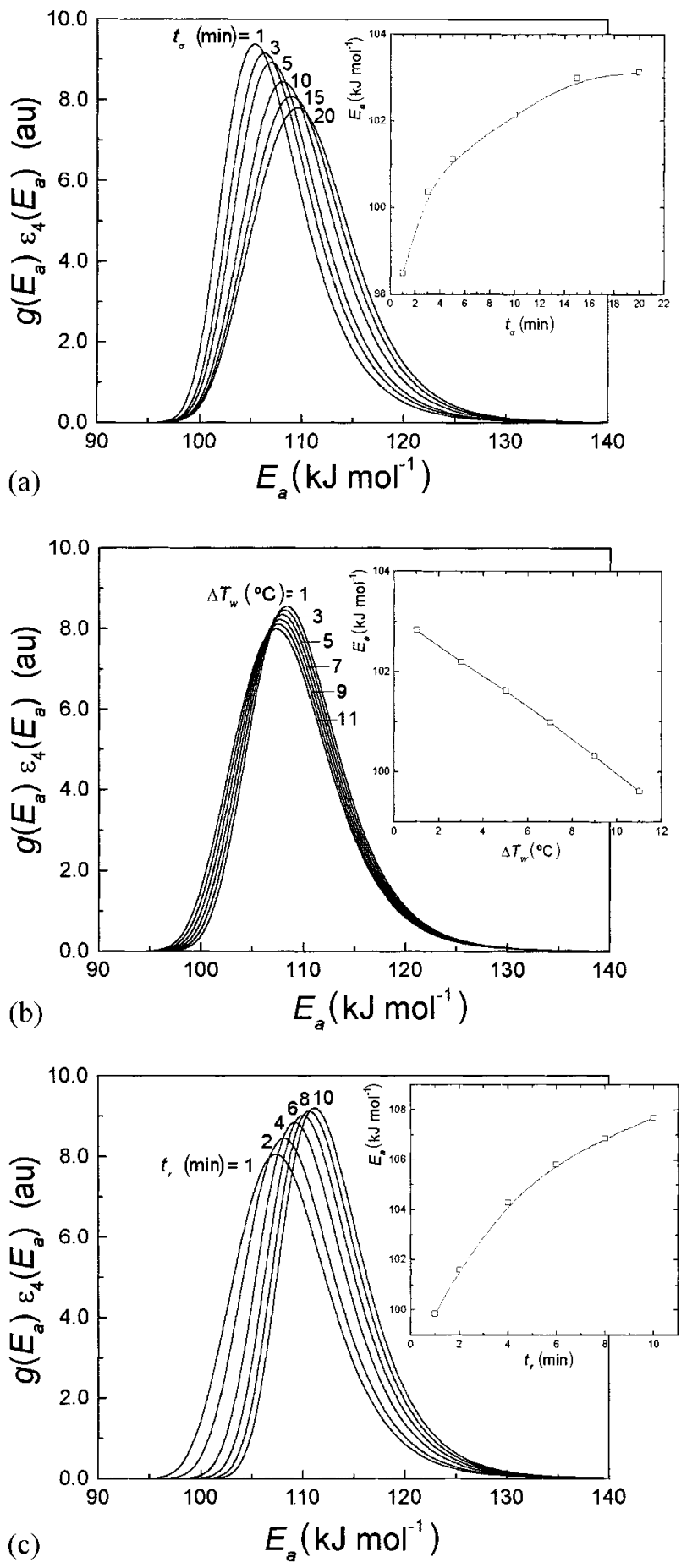

Figure 4. Distribution of the activation energy by the species that contribute to the strain at the beginning of step 5(:) (a) influence of $t_{\sigma}$ (simulated data shown in Fig 2(a); (b) influence of $\Delta T_{\mathrm{w}}$ (simulated data in Fig 2(b); (c) Influence of $t_{\mathrm{r}}$ (simulated data in Fig 2(c). Inset graphics show activation energy as a function of the varied parameter. 
higher values, whereas with the increase of $\Delta T_{\mathrm{w}}$ or the decrease of $t_{\mathrm{r}}$ the intensity of the peaks increases and the peaks shift to lower temperatures. Previous studies reported the influence of $\Delta T_{\mathrm{w}}$ in TSDC results ${ }^{15,16}$. As observed in the present work, the TS peaks from TSDC results on a liquid crystalline polymer shift to lower temperatures and are more intense when $\Delta T_{\mathrm{w}}$ increases. $^{16}$

Figure 5(b) seems to shows that, experimentally, $\Delta T_{\mathrm{w}}$ is much more effective in changing the intensity of
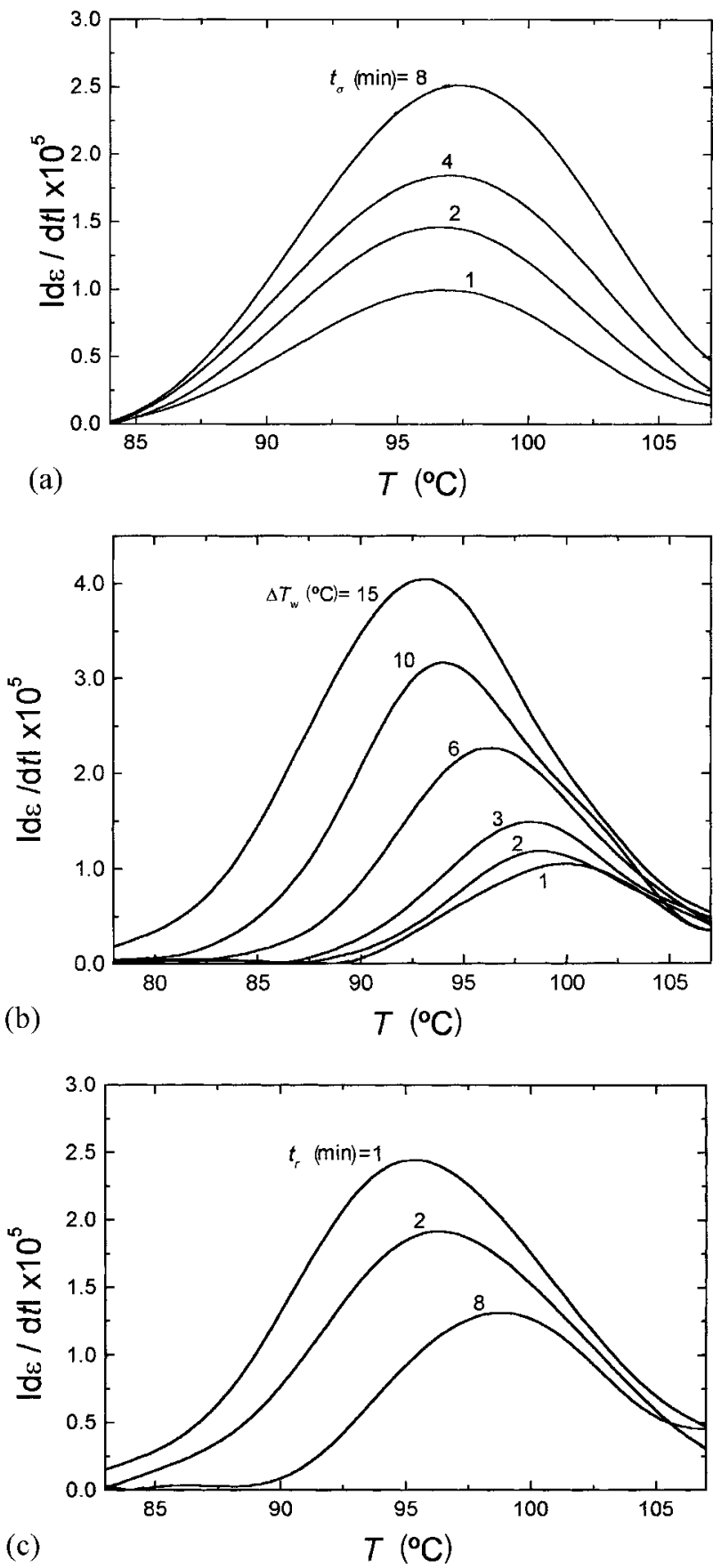

Figure 5. Experimental TS results obtained with the studied PET film at $T_{\sigma}=93^{\circ} \mathrm{C}$; (a) influence of $t_{\sigma}$ (shown in the graphics) the other experimental conditions being $\Delta T_{\mathrm{w}}=4^{\circ} \mathrm{C}$ and $t_{\mathrm{r}}=2 \mathrm{~min}$; (b) influence of $\Delta T_{\mathrm{w}}$ (shown in the graphics) the other experimental conditions being $t_{\sigma}=4 \mathrm{~min}$ and $t_{\mathrm{r}}=4 \mathrm{~min}$; (c) influence of $t_{\mathrm{r}}$ (shown in the graphics) the other experimental conditions being $t_{\sigma}=4 \mathrm{~min}$ and $\Delta T_{\mathrm{w}}=4^{\circ} \mathrm{C}$. the peaks than in the simulated results (Fig 2(b)). However, one must be cautious with such an analysis. It is not possible to compare the simulated results and the experimental data directly because a distribution of activation energies was used in the simulations, whereas in the glass transition region of polymers a combination of a distribution of both activation energies and pre-exponential factors is assigned to the spectrum of the retardation times. ${ }^{17,18}$ In fact, this leads to a different response in the TS curves that is, for example, clearly shown by the shape of the resulting peaks: the simulated peaks are broader and more asymmetrical than the experimental ones. One should then try to maintain the discussion about comparing the tendencies of the features of the peaks to vary when the experimental variables used are changed.

The Arrhenius lines were obtained for the experiments shown in Fig 6 (inset graphics). The main graphics of Fig 6 show the variation of the activation energy against the studied variable. A decrease of activation energy is observed with increasing creep time $t_{\sigma}$ (Fig 6(a)). This tendency is opposite to that observed with the simulation results (Fig 4(a)). This interesting behaviour may be explained by the fact that $T_{\sigma}$ is close to but below $T_{\mathrm{g}}$. Therefore, the material is affected by structural relaxation during its creep period. Structural relaxation designates the process of approaching an equilibrium state undergone by a glass held at constant environmental conditions after its formation history. ${ }^{19,20}$ It has been shown that thermally stimulated depolarization currents undergo changes when they experience different thermal histories below $T_{\mathrm{g}}{ }^{21-25}$ Also, changes in the TS results were observed by TSR, during structural relaxation. ${ }^{26}$ In that study a clear decrease of activation energy was detected with increasing ageing time at $T_{\sigma}$ before the TS experiment. This tendency for $E_{\mathrm{a}}$ to decrease with ageing may be more important than the tendency of this parameter to increase with increasing $t_{\sigma}$, as detected in the simulation results. Note that in the simulations the structural relaxation phenomenon is not considered.

As in the simulations (inset in Fig 4(b)), the experimental results show a decrease of $E_{\mathrm{a}}$ with increasing $\Delta T_{\mathrm{w}}$ (Fig 6(b)). The same tendency was previously observed in TSDC results obtained for a fluorinated copolymer at different polarization temperatures in the glass transition region. ${ }^{15}$ It is interesting to observe the Arrhenius lines (inset in Fig 6(b)): a tendency of increasing curvature in the lines is seen as $\Delta T_{\mathrm{w}}$ increases. This is a consequence of the broadening of the retardation time distribution that is stored in the sample as $\Delta T_{\mathrm{w}}$ increases.

The influence of the recovery time $t_{\mathrm{r}}$ on the experimental results (Fig 6(c)) is also in agreement with the simulations (Fig 4(c)). In both cases $E_{\text {a }}$ increases with $t_{\mathrm{r}}$ in a similar manner. As in the variation of $t_{\sigma}$ (Fig 6(a)), no apparent variation of the 
curvature of the Arrhenius lines is observed when $t_{\mathrm{r}}$ is changed.

Figure 6 shows that the modification of the experimental variables of the TS programmes changes the obtained thermokinetic parameters greatly, namely
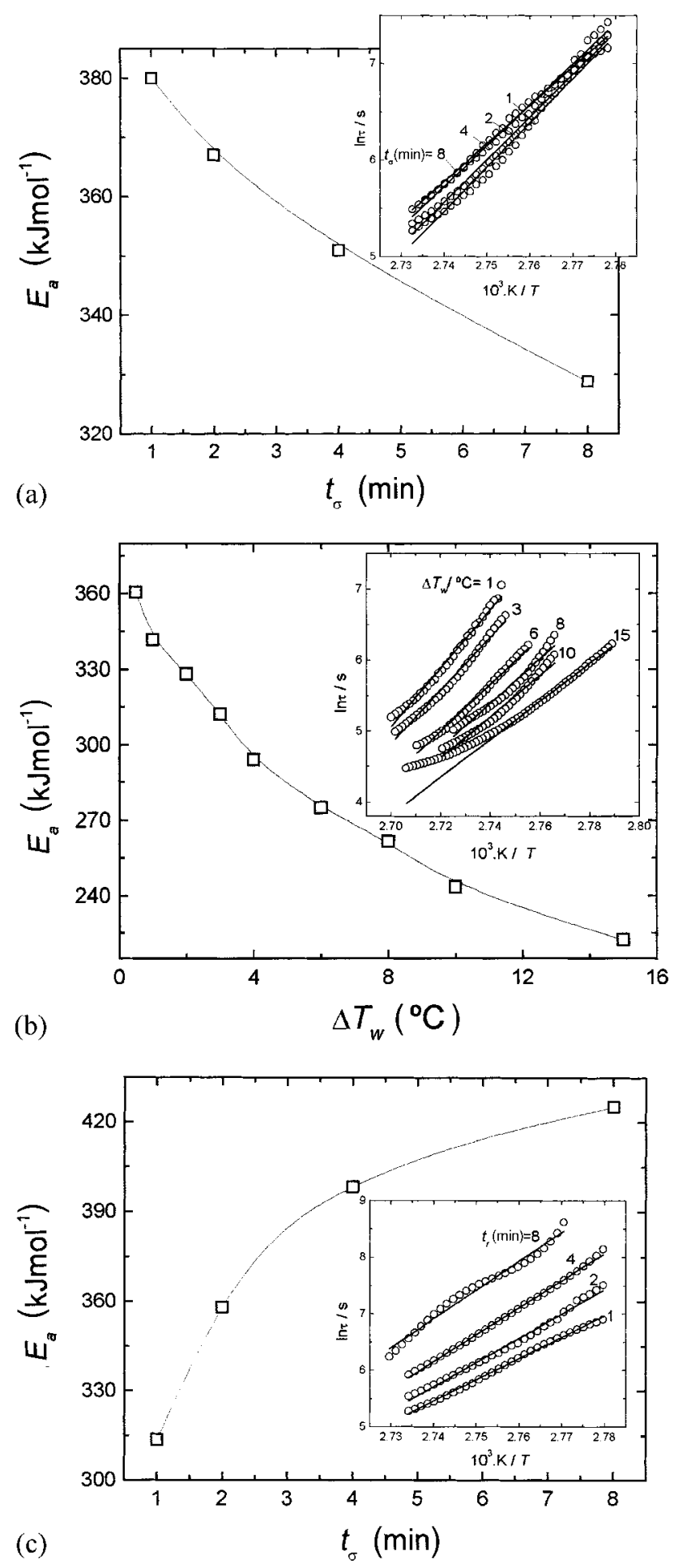

Figure 6. Activation energies of the TS curves obtained according to the experimental procedures presented in Fig 5. Inset graphics show Arrhenius lines of the experimental data (obtained using eqn (9), from which the activation energies were calculated. Note that in part (b), the activation energy of the Arrhenius line for the experiment at $\Delta T_{\mathrm{w}}=15^{\circ} \mathrm{C}$ was calculated neglecting the high temperature region, due to the high curvature observed. the activation energy. In fact, in the studied range of the $t_{\sigma}$ values we detected a variation of about $50 \mathrm{~kJ}$ $\mathrm{mol}^{-1}$. Such a variation is even more pronounced for the influence of $\Delta T_{\mathrm{w}}$ and $t_{\mathrm{r}}$ (about $115 \mathrm{~kJ} \mathrm{~mol}^{-1}$ and $110 \mathrm{~kJ} \mathrm{~mol}^{-1}$, respectively). It should be noted that the experimental errors associated with the determination of $E_{\mathrm{a}}$ are of the order of $5-10 \%,{ }^{12}$ which is considerably below the observed changes in $E_{\mathrm{a}}$ with the changing of the experimental variables. The range of activation energies obtained from a certain variation of one parameter depends on $T_{\sigma}$ (or the polarization temperature for the case of the TSDC technique), as was reported for the influence of $\Delta T_{\mathrm{w}} \cdot{ }^{15}$ When one increases $T_{\sigma}$, upon approaching $T_{\mathrm{g}}$, the range of the calculated activation energies increases for the same variation of $\Delta T_{\mathrm{w}}$. Teyssèdre and Lacabanne ${ }^{15}$ report that differences of $E_{\mathrm{a}}$ for variations of $\Delta T_{\mathrm{w}}$ between 0 and $10^{\circ} \mathrm{C}$ may exceed $130 \mathrm{~kJ} \mathrm{~mol}^{-1}$ for high polarization temperatures (near $T_{\mathrm{g}}$ ). Note that in this work the $T_{\sigma}$ used was quite close to $T_{\mathrm{g}}{ }^{27}$ which justifies the great variation detected in $E_{\mathrm{a}}$.

To characterize a complex relaxation, TS experiments are usually performed at different $T_{\sigma}$ (or polarization temperature for TSDC). A typical feature near $T_{\mathrm{g}}$ is the occurrence of the compensation phenomenon that corresponds to a linear relationship between $E_{\mathrm{a}}$ and $\log \tau_{0}$ of the different TS curves (see, for example, Moura Ramos et $a l^{28}$ and references cited therein). A typical example is shown in Fig 7, where the thermokinetic parameters of some TS experiments on the studied PET carried out at different $T_{\sigma}$ are shown as black squares.

Besides the activation energies, the pre-exponential factor $\tau_{0}$ is also influenced by the studied experimental parameters. In Fig 7 the two Arrhenius parameters of all the TS experiments are shown against each other. A perfect linear relationship is observed (solid line in Fig

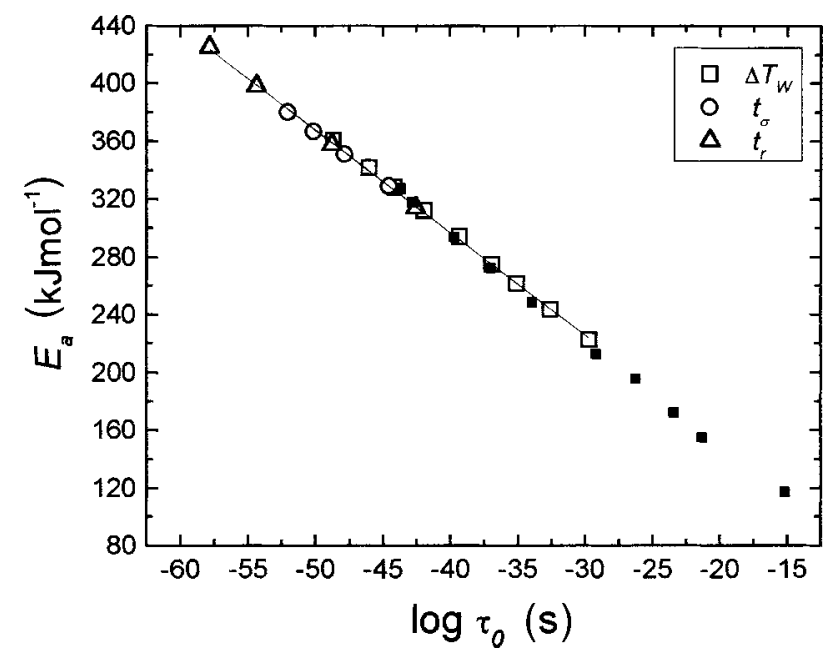

Figure 7. Relationship between the Arrhenius parameters of the TS results obtained by changing the different experimental parameters: $t_{\sigma}$ (circles), $\Delta T_{\mathrm{w}}$ (squares) and $t_{\mathrm{r}}$ (triangles). The solid line corresponds to the linear fitting of the open points. The black squares are for TS experiments performed on the same PET at varied $T_{\sigma}$ (also in the glass transition region) and at the same $t_{\sigma}, \Delta T_{\mathrm{w}}$, and $t_{\mathrm{r}}$. 
7), indicating a high correlation between both parameters, which is independent of the variable that is changed. It must be noted that such linearity (that cannot have a trivial correlation with the mobility at the molecular level) is very similar to that of the typical compensation effect shown by the black squares (experiments obtained at different $T_{\sigma}$ ). This is a clear indication of the difficulty of assigning a true microscopic physical origin to the compensation phenomenon, as presented in many TSR or TSDC works.

It must be noted finally that for a more detailed comparison between simulations and data near $T_{\mathrm{g}}$ a more realistic temperature dependence of the characteristic time and the corresponding distribution should be considered that could take into account the nonlinear and non-exponential features of the glass transition. ${ }^{19,20}$ Such work, that extends the main goals of the present study, would allow a more quantitative investigation of the influence of the structural relaxation in the TSR or TSDC results and would provide more general insights on some issues related to these techniques. For example, one could check if the compensation effect is a natural event assigned to the techniques itself or whether it is a result of the propagation of experimental errors.

\section{CONCLUSIONS}

The use of a simple model to describe the TSR experiments on a system characterized by a distribution of activation energies allows the following variations of the TS peaks with $t_{\sigma}, \Delta T_{\mathrm{w}}$ and $t_{\mathrm{r}}$ to be predicted: (i) an increasing intensity of the TS peaks and a shift to higher temperatures as $t_{\sigma}$ increases; (ii) an increasing intensity of the TS peaks and a shift to lower temperatures as $\Delta T_{\mathrm{w}}$ increases or $t_{\mathrm{r}}$ decreases.

The same variations of the studied parameters were also detected in experimental TS results for a semicrystalline PET in its glass transition region. For this particular case it was concluded that the decrease of $\Delta T_{\mathrm{w}}$, usually considered to be the more obvious procedure by which to narrow the distribution of retardation times, is not the most efficient way to isolate a quasi-elementary process. The simulations allowed the narrowing efficiency of the distribution of retardation times during a TS experiment to be investigated by looking at the distribution of activation energies of the system before the final heating. For the particular system studied, the decrease of $t_{\sigma}$ appears to be the most efficient strategy. As in the simulations, the activation energy of the experiments decreases as $\Delta T_{\mathrm{w}}$ increases or as $t_{\mathrm{r}}$ decreases. However, the effect of $t_{\sigma}$ on the activation energy is opposite to that obtained with the modelling: in the experiments a decrease in the activation energy is observed as $t_{\sigma}$ increases. This may be a consequence of the structural relaxation undergone by the sample during the creep period because $T_{\sigma}$ is some degrees below $T_{\mathrm{g}}$, a phenomenon not considered in the model used.

It was shown that the change of any of the studied experimental parameters could significantly affect the response of a material studied by TSR. In particular, the modification in the thermokinetic parameters (namely the activation energy) of the TS experiments may be very pronounced, especially if $T_{\sigma}$ is close to but below $T_{\mathrm{g}}$. The conclusions of this work should also be applicable to the TSDC technique.

The broad range of $E_{\mathrm{a}}$ or $\log \tau_{0}$ covered by TS experiments when one changes experimental parameters, as was seen in Figs 6 and 7, suggests that it is not trivial to attribute a certain value of $E_{\mathrm{a}}$ to an experiment carried out at a certain $T_{\sigma}$. To be coherent, the experiments performed on a material at different $T_{\sigma}$ (or different polarization temperatures in TSDC) should use the same $t_{\sigma}, \Delta T_{\mathrm{w}}$ and $t_{\mathrm{r}}$ values. The same should apply when the purpose is to compare different materials.

\section{ACKNOWLEDGEMENTS}

NMA and JFM acknowledge the financial support of Fundação para a Ciência e Tecnologia (Project PRAXIS/P/CTM/14171/1998). Support from the Portuguese-Spanish joint research action (Spain: HP1999-0024; Portugal: E/69/00) is also acknowledged.

\section{REFERENCES}

1 van Turnhout J, Thermally Stimulated Discharge of Polymer Electrets, Elsevier, New York (1975).

2 Lavergne C and Lacabanne C, IEEE Electr Insul Mag 9:5 (1993).

3 Mano JF, Correia NT and Moura Ramos JJ, Polymer 35:3561 (1994).

4 Sauer BB and Kim YH, Macromolecules 30:3323 (1997).

5 Doulut S, Demont P and Lacabanne C, Macromolecules 33:3425 (2000).

6 Chatain D, Gautier PG and Lacabanne C, f Polym Sci 11:1631 (1972).

7 Lacabanne C, Chatain DC and Mompajens JC, $\mathcal{F}$ Macrom of Sci Phys B 134:537 (1977).

8 Lacabanne C, Chatain DC, Mompajens JC, Hiltner A and Baer E, Solid State Commun 27:1055 (1978).

9 Lacabanne C, Lamure A, Teyssedre G, Bernès A and Mourgues M, J Non-Cryst Solids 172-174:884 (1994).

10 Alves NM, Mano JF and Gómez Ribelles JL, Macromol Symp 148:437 (1999).

11 Mano JF, f Phys D Appl Phys 31:2898 (1998).

12 Alves NM, Mano JF and Gómez Ribelles JL, submitted for publication.

13 Zielinski M, Swiderski T and Kryszewski M, Polymer 19:883 (1978).

14 Mano JF, f Phys D Appl Phys 33:280 (2000).

15 Teyssèdre G and Lacabanne C, f Phys D Appl Phys 28:1478 (1995).

16 Correia NT and Moura Ramos JJ, f Polym Sci Polym Phys Ed 37:227 (1999).

17 Pelster R, Kruse T, Krauthäuser HG, Nimtz G and Pissis P, Phys Rev B 57:8763 (1998).

18 Pelster R, Kruse T, Krauthäuser HG, Grunow V, Nimtz G and Pissis P, F Non-Cryst Solids 235-237:160 (1998).

19 Hodge IM, f Non-Cryst Solids 169:211 (1994).

20 Hutchinson JM, Prog Polym Sci 20:703 (1995).

21 Doulut $S$, Becharan C, Demont P, Bernès A and Lacabanne C, $\mathcal{f}$ Non-Cryst Solids 235-237:645 (1998).

22 Belana J, Canãdas JC, Diego JA, Mucharra M, Diaz R, 
Friederichs S, Jaimes C and Sanchis MJ, Polym Int 46:29 (1998).

23 Cañadas JC, Diego JA, Mudarra $\mathrm{M}$ and Belana J, Polymer 39:2795 (1998).

24 Diego JA, Canãdas JC, Mudarra $M$ and Belana J, Polymer 40:5355 (1999).
25 Correia NT, Alvarez C and Moura Ramos JJ, f Chem Phys 113:3204 (2000).

26 Alves NM, Mano JF and Gómez Ribelles JL, Polymer 42:4173 (2001).

27 Alves NM, Mano JF and Gómez Ribelles JL, accepted.

28 Moura Ramos JJ, Mano JF and Sauer BB, Polymer 38:1081 (1997). 